\title{
Ultrastructure des cellules de Sertoli humaines aux stades embryonnaires précoces de la différenciation gonadique
}

\author{
S. MAKABE*, R. HeYN ${ }^{* *},{ }^{*}$, P.M. MotTA ${ }^{\circ}$ \\ *Département de Gynécologie-Obstétrique. Ecole de Médecine de l'Université de Tokio, \\ Japon; **Département de Morphologie Expérimentale, Université du Chili, Santiago du \\ Chili ; Département d'Anatomie. Faculté de Médecine, Université de Rome "La Sapienza", \\ Via Alfonso Borelli, 50, 00161 Rome, Italie.
}

\section{RESUME}

Le développement morphofonctionnel des cellules de Sertoli détermine la différenciation testiculaire. Ces cellules somatiques sont pour la plupart d'origine mésonéphrotique et peuvent être au plus tôt reconnues chez les embryons de 7 semaines lorsque se constituent les cordons testiculaires. Ces derniers s'organisent à partir des cellules germinales primordiales entourées par les cellules présertoliennes. En rapport avec la grande activité de synthèse des cellules présertoliennes, le reticulum endoplasmique granuleux se développe. La lamina basale des cordons devient visible vers la 7ème, 8ème semaine du développement. Prespermatogonies et cellules présertoliennes prolifèrent activement mais ces dernières sont beaucoup plus nombreuses que les spermatogonies. On observe de nombreux interdigitations et processus cytoplasmiques entre les cellules présertoliennes voisines. En raison de l'activité proliférative il s'établit une sorte de compartimentation à l'intérieur des cordons dans lesquels les cellules présertoliennes tendent à se localiser plus près de la membrane basale, entourant les préspermatogonies par des processus cytoplasmiques longs et minces. L'une des principales caractéristiques des cellules présertoliennes en différenciation est leur noyau irrégulier à volumineux nucléole. Chez l'embryon de 14 à 20 semaines, le changement le plus significatif est représenté par le développement maximum des cellules de Leydig. Les cordons testiculaires ne possèdent pas de lumière et ne peuvent être appelés "tubules".

Mots clés : Cellules de Sertoli, testicule, embryogenèse humaine, MEB, MET.

\section{INTRODUCTION}

A la fin de la 6ème semaine du développement les tractus génitaux des embryons mâles et femelles ne peuvent apparemment pas être distingués, sauf par de subtiles différences cellulaires. Les cordons sexuels et les cellules germinales primordiales sont déjà présents à la fois dans les régions corticale et médullaire de la gonade indifférenciée. Cependant le stade dit "indifférent" du développement de la gonade est arrivé à son terme. En fait, à partir de la 7ème semaine du développement la différenciation masculine est stimulée par un facteur spécifique synthétisé dans les cordons (le "testis-determining factor - TDF - codé par le gène responsable de la différenciation sexuelle localisé sur le chromosome $\mathrm{Y}^{\text {“) }}$ [9, $15,16]$. 
On a pu dire que la différenciation de cellules somatiques en cellules de Sertoli témoigne du début de la formation du testicule. De fait, pendant la 7ème semaine, la différenciation testiculaire commence avec la différenciation des cellules de Sertoli qui, dans le même temps, établissent des contacts étroits entre elles et avec les cellules germinales primordiales (gonocytes primordiaux) pour former les cordons testiculaires $[2,8-10,15,16,22]$.

Les cellules présertoliennes correspondent à des cellules somatiques qui proviennent surtout des régions mésonéphrotique $[2,8$, $16,26)$ et cœlomique $[2,10,26]$ de la gonade et prolifèrent pendant tout le développement fœetal. Ces cellules sécrètent de l'hormone antimüllérienne (AMH) et une substance inhibitrice des canaux de Müller (MIS) peu après leur agrégation dans les cordons testiculaires. L'AMH est une glycoprotéine de la famille du TGFb [7-9, 15]. Dans ces conditions, le contact entre les cellules présertoliennes et les gonocytes primordiaux jouerait un rôle clé dans la différenciation sexuelle masculine en contrôlant la prolifération et le développement précoces des cellules germinales $[9,10,14,15]$. Ainsi que nous l'avons montré dans de précédentes publications $[11,12]$ la méthode à l'Osmium-DMSO-Osmium (ODO) est la technique la plus efficace pour la mise en évidence des organites cellulaires et en combinaison avec la microscopie électronique à balayage (MEB) à haute résolution, elle permet d'obtenir des images originales de la micro-architecture tridimensionnelle du testicule [12].

Le but de la présente étude est de faire le point de nos connaissances et d'apporter de nouvelles informations sur l'ultrastructure du testicule humain, en considérant particulièrement les cellules de Sertoli entre la 6ème et la 20ème semaine du développement. Des échantillons sélectionnés et traités par la méthode de macération à l'ODO ont été observés en MEB à haute résolution et, parallèlement, en microscopie électronique à transmission (MET).

\section{MATERIEL ET METHODES}

Des testicules humains appartenant à des embryons de $6,7,7$ à $8,14,16$ et 20 semaines de développement et provenant d'avortements spontanés ou d'interventions chirurgicales (hysterectomie, hysterotomie) ont été découpés immédiatement après leur prélèvement. Toutes les patientes ont été informées du protocole et ont donné leur consentement. L'âge foetal a été estimé en mesurant les longueurs vertex-talon, ou vertex-bourgeon caudal $[4,17]$ et à partir du dernier cycle maternel. Dans les stades les plus précoces le sexe a été déterminé par caryotype.

\section{Microscopie électronique à trans- mission (MET)}

Les échantillons ont été fixés dans le glutaraldéhyde à $2,5 \%$, post fixés avec une solution aqueuse de tétroxyde d'osmium à $1,33 \%$ pendant 2 heures, déshydratés dans des bains à concentration croissante d'éthanol et inclus dans l'Epon 812. Des coupes minces ont été effectuées avec des ultra microtomes LKB II, Porter-Blum MT1 ou Ultracut E.Reichert-Jung, montées sur des grilles sans support et colorées par l'acétate d'uranyl et le citrate de plomb. Les coupes ont été examinées et photographiées avec des microscopes électroniques Zeiss EM 9A et Philips EM 400.

\section{Microsocopie photonique (MP)}

Des fragments des échantillons étudiés en MET ont été l'objet de coupes sériées de 1 $\mu \mathrm{m}$ d'épaisseur, colorées au bleu de toluidine et photographiées avec un microscope Zeiss Ultraphot II. Les résultats obtenus en MP ont été utilisés pour définir l'orientation des cellules et tissus et ont été intégrés avec les observations parallèles en MEB et MET. 


\section{Microscopie électronique à balayage (MEB)}

Les échantillons ont été fixés par immersion dans le glutaraldéhyde à $2,5 \%$, post fixés dans une solution aqueuse de tétroxyde d'osmium à $1,33 \%$ pendant 2 heures et déshydratées dans plusieurs bains d'éthanol. Les gonades ont été désséchées jusqu'au point critique en utilisant du gaz carbonique liquide (Balzers Union CPD 020 critical point dryer), montées sur support d'aluminium avec de la pâte d'argent et recouverts de platine dans un appareil $\mathrm{K} 55 \mathrm{O}$ sputter coater (EMITECH). Les observations ont été effectuées avec un MEB Hitachi S4000 à émission de champ opérant à un voltage accélérant de 7 à $10 \mathrm{kV}$.

\section{MEB + Technique de macération ODO}

Les échantillons ont été fixés par immersion dans le glutaraldhéhyde à $0,5 \%$ plus de la paraformaldhéhyde à $0,5 \%$ dans un tampon phosphate $M / 15$, post fixés dans le tétroxyde d'osmium à $1 \%$ dans un tampon phosphate $\mathrm{M} / 15$ pendant 1 à 2 heures, rincés avec la même solution tampon et ensuite immergés dans des solutions aqueuses de diméthyl-sulfoxyde (DMSO) à $15 \%, 30 \%$ et $50 \%$ pendant 30 minutes pour chaque bain, dans le but de prévenir les dommages causés par les cristaux de glace au cours de la congélation effectuée à l'étape suivante. Utilisant la technique précédemment décrite $[11,21]$, les échantillons ont été congelés sur des plaques métalliques refroidies par l'azote liquide, brisés en deux parties avec une lame de rasoir et un marteau et immédiatement placés dans une solution aqueuse de DMSO à 50\%. Après rinçage dans la solution tampon, les échantillons ont été placés dans un bain de tétroxyde d'osmium à $0,1 \%$ dans un tampon phosphate $\mathrm{M} / 15$ à $20^{\circ} \mathrm{C}$ pendant 1 à 3 jours et fixés à nouveau pendant 1 heure dans le tétroxyde d'osmium à $1 \%$ dans la même solution tampon. Les échantillons ont été ensuite colorés par immersion dans une solution aqueuse à $2 \%$ d'acide tannique pendant 1 heure puis dans le tétroxyde d'osmium à $1 \%$ dans la même solution tampon pendant 1 heure. Après déshydratation dans des bains d'éthanol de concentrations graduées et traitement par l'acétate d'isoamyl, les échantillons ont été désséchés au point critique et recouverts d'une couche d'environ $3 \mathrm{~nm}$ de platine dans un appareil à pulvérisation ionique muni d'un dispositif rotatif (VX-10R, Eiko Engineering Co, Ltd, Japan) et observé avec un MEB à émission de champ (HFS2ST, Hitachi CO, Ltd, Japan).

\section{RESULTATS ET DISCUSSION}

Depuis le travail original de Sertoli (Dell'esistenza di particulari cellule ramificate nei canalicoli seminiferi del testicolo umano. Morgagni $7: 31-39,1865$ ) et les dessins de Von Eberhard [23], peu d'études ont été entreprises en ce qui concerne le développement des cellules de Sertoli. D'ailleurs les travaux de Fukuda et al. [5], Satoh [20] et Wartenberg et al. [25], tout en montrant d'élégantes images des cordons testiculaires à différents âges, ne mentionnnent pas du tout les cellules de Sertoli.

A la 6ème semaine du développement, le blastème gonadique est constitué de gonocytes primordiaux et de cellules mésenchymateuses. Ces dernières sont de forme irrégulière, avec un cytoplasme peu développé contenant des mitochondries en bâtonnets denses aux électrons. Les gonocytes primordiaux se distinguent nettement des cellules mésenchymateuses en raison de leur forme sphérique et de leurs grands noyaux arrondis (Figure 1) $[6,10]$.

Les cellules somatiques qui sont à l'origine des cellules présertoliennes s'agrégent et tendent à définir un contour régulier annonçant la surface du futur cordon testiculaire [10]. Dès que les premières cellules présertoliennes se réunissent, elles se séparent des cellules mésenchymateuses voisines (Figure 2). Les cellules présertoliennes typiques sont pauvres en organites cytoplasmiques représentés par des mito- 


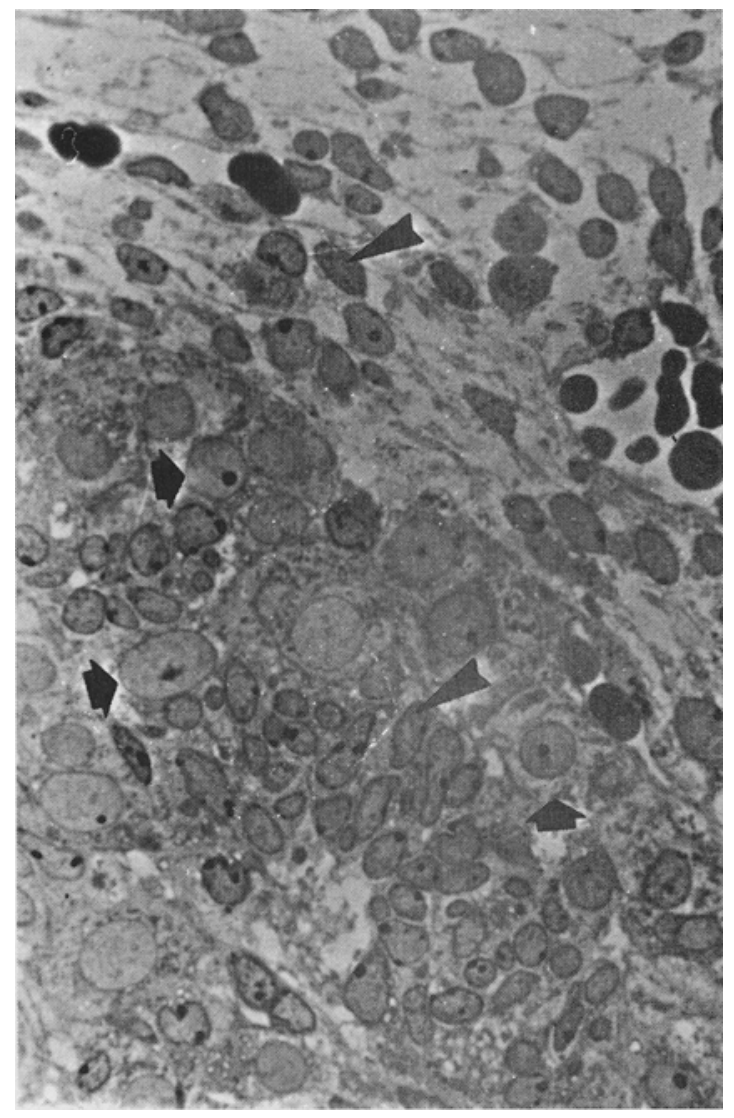

Figure 1 : Gème semaine de développement. La gonade indifférenciée contient des masses de cellules qui ne sont pas encore organisées en cordons testiculaires. Les cellules germinales primordiales (flèches) et les cellules mésenchymateuses (têtes de flèches) sont reconnnaissables. Microscope photonique, hématoxyline-éosine, $198 \mathrm{X}$.

chondries en bâtonnets fortement colorées et disposées autour des noyaux en colonnes (Figures 2-4) [6, 13, 24]. En fait, elles commencent à envelopper les gonocytes primordiaux maintenant appelés "préspermatogonies". Par conséquent, à 7 semaines de développement, les cordons testiculaires sont clairement reconnaissables, même en microscopie photonique (Figures 2 et 4 ). Aucune membrane basale n'est visible autour des cellules présertoliennes pendant les stades initiaux de la formation des cordons testiculaires [10]. Cependant, à 7 semaines de développement on peut mettre

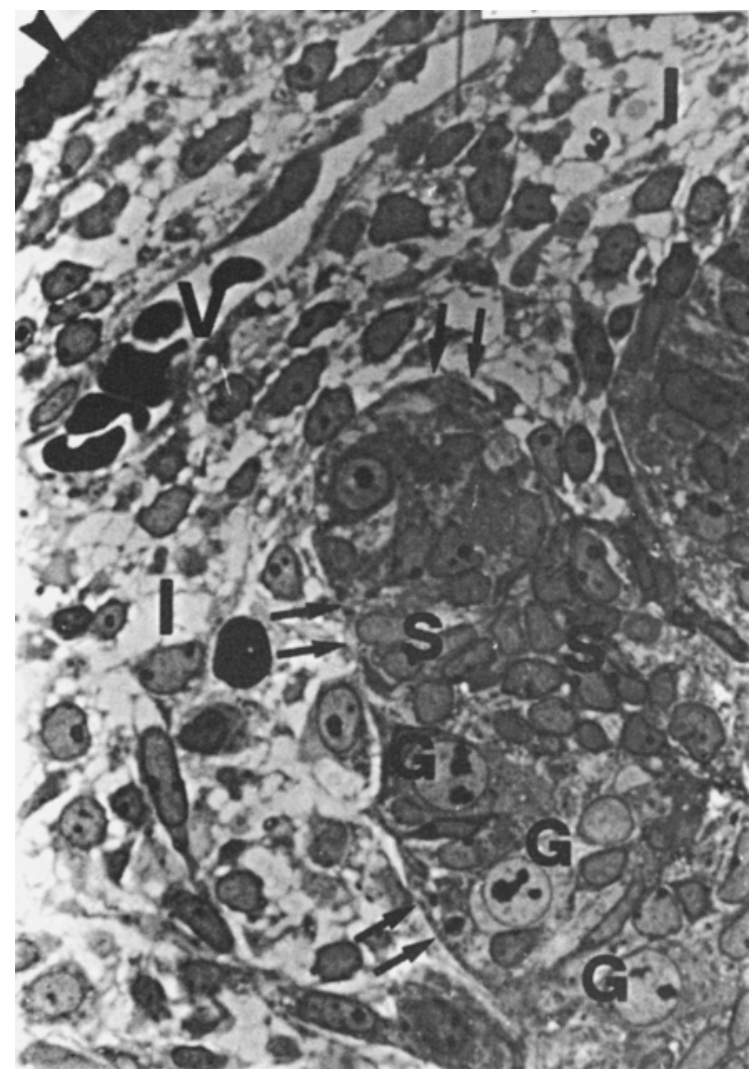

Figure 2 : 7ème semaine de développement. Epithélium colomique (tête de flèche). Les cordons ramifiés (flèches) sont clairement délimités par des laminae basales et contiennent des préspermatogonies (G) et des cellules présertoliennes (S). Les cellules mésenchymateuses, ainsi que les cellules germinales primordiales en migration, constituent l'interstitium (I). V : vaisseaux. Microscope photonique, hématoxyline-éosine, $198 X$.

en évidence des laminae qui délimitent les cordons (Figure 2) [17, 20, 22].

Chez l'embryon âgé de 7 à 8 semaines le nombre des cellules présertoliennes dépasse largement celui des préspermatogonies en raison d'une intense activité mitotique entraînant leur multiplication jusqu'au début de la spermotogenèse [2, 13]. En conséquence de cette prolifération cellulaire les noyaux des cellules présertoliennes ont parfois un aspect comprimé (Figure 5) $[2,6$, $10]$. 


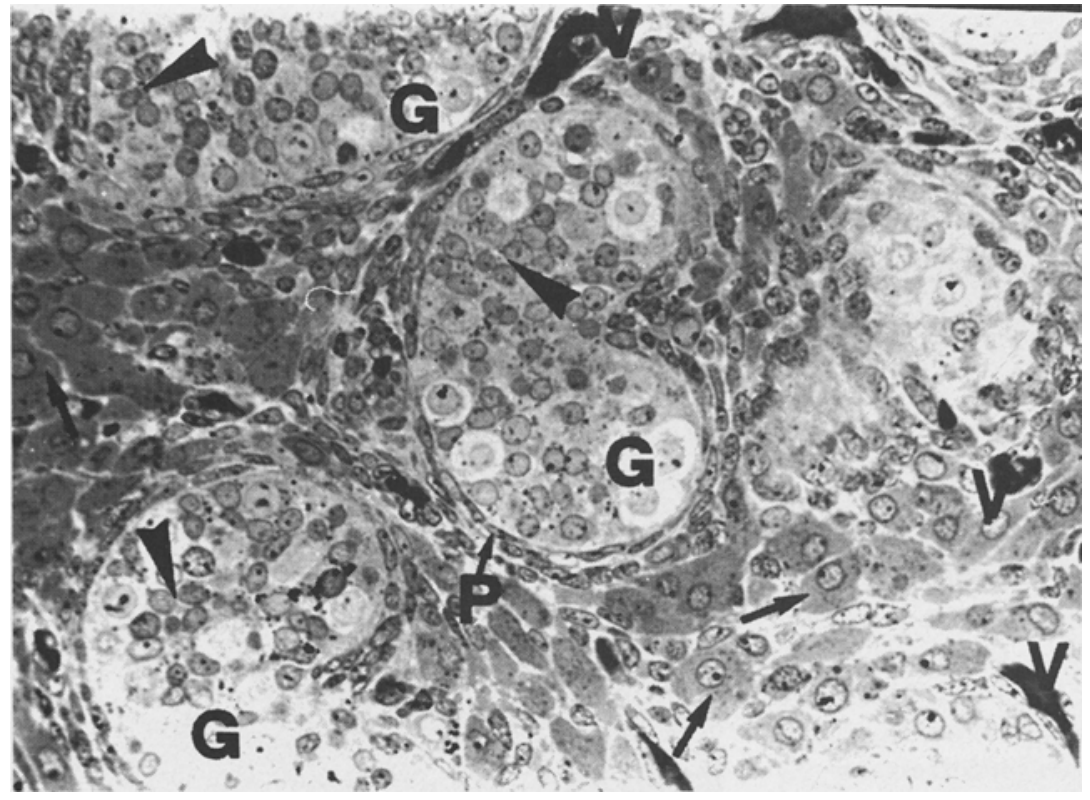

Figure 3 : 16ème semaine de développement. L'interstitium contient de nombreuses cellules de Leydig (flèches). G : préspermatogonies. Têtes de flèches : cellules présertoliennes. $P$ + flèches : futures cel. lules péritubulaires. $V$ : Vaisseaux. Microscope photonique, bleu de toluidine, $200 \mathrm{X}$.

On sait que les cellules présertoliennes produisent différentes protéines telles que l'AMH, la desmine, la cytokératine et la transferrine [7, 17, 18]. En fait, les empilements de lamelles parallèles du reticulum endoplasmique granuleux et les mitochondries globulaires deviennent plus abondants (Figures 4 et 5) [1, 2, 4, 6, 13] ; ces deux éléments sont caractéristiques des cellules en différenciation. De plus, les nucléoles deviennent clairement visibles (Figure 5) $[4,13]$ et des corps denses, probablement des gouttelettes lipidiques, peuvent être observés (Figures 5 et 7).

Progressivement la forme des cellules présertoliennes devient de plus en plus irrégulière avec la présence d'interdigitations et de replis membranaires, parfois minces et étendus, pénétrant dans les cellules présertoliennes voisines (Figures 5 et 6$)[2,8,10]$. Ces processus cytoplasmiques forment souvent un système extrêmement complexe dans lequel les préspermatogonies sont séparées par des cordons de cytoplasme des cellules présertoliennes et n'entrent qu'occasionnellement en contact les unes avec les autres [6]. Ces processus jouent probablement un rôle dans l'incorporation des

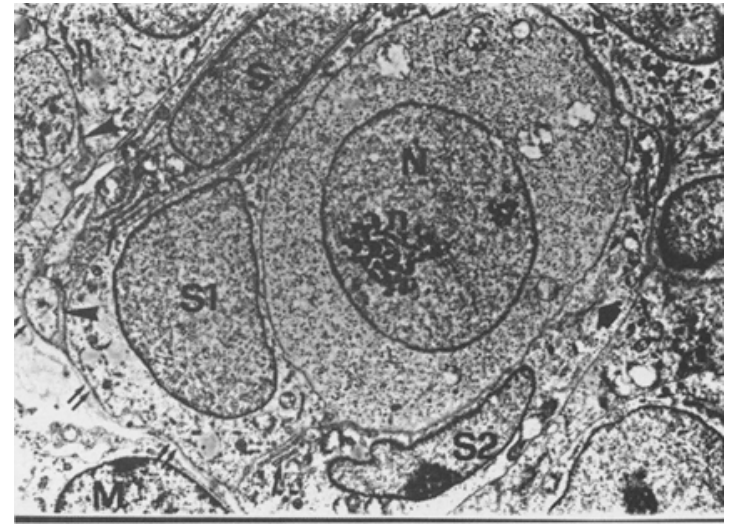

Figure 4 : Section transversale de cordon testiculaire à 7 semaines de développement. Une cellule germinale primordiale est entourée par des cellules présertoliennes (S). La première possède un noyau régulièrement arrondi $(N)$ avec une chromatine finement granuleuse et un nucléole filiforme (n); Des rares mitochondries contiennent des crêtes dilatées et sont situées près du noyau. Les cellules présertoliennes possèdent soit un noyau ovoüde régulier (1) soit un noyau irrégulier plus différencié (2). A noter le reticulum endoplasmique granuleux bien développé (flèches) et l'appareil de Golgi (flèche large). Têtes de flèches: interdigitations. Doubles flèches : lamina basale. M : mésenchyme indifférencié. MET, X 3000. 


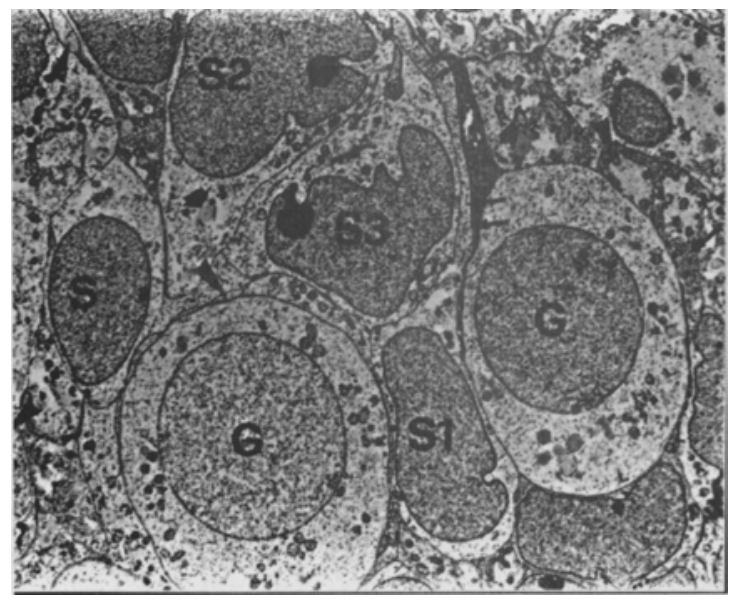

Figure 5 : Section transversale d'un cordon testiculaire entre la 7ème et la 8ème semaine du développement. En raison de l'intense activité proliférative des préspermatogonies (G) et des cellules présertoliennes (S) les noyaux de ces dernières paraissent parfois comprimés (S1). A noter la présence de quelques cellules présertoliennes plus différenciées avec des noyaux indentés et de volumineux nucléoles (S2 et S3) ainsi que de longs processus cytoplasmiques (doubles flèches). Les flèches larges indiquent des corps denses (probablement des lipides) et les flèches montrent le reticulum endoplasmique granuleux dans les cellules présertoliennes. Têtes de flèches : interdigitations. MET, $X 2500$.

gonocytes primordiaux dans les cordons testiculaires [10] et dans la compartimentation initiale des cordons, base fonctionnelle de la future organisation de la barrière hémo-testiculaire.

Les cellules présertoliennes sont, soit adjacentes au tissu qui entoure les cordons, soit diposées dans une situation plus centrale en raison de l'entassement des cellules à l'intérieur des cordons [6, 17]. Elles n'ont pas encore développé les complexes de jonction qui constitueront plus tard la barrière hémo-testiculaire chez l'adulte [2]. Cependant, quelques jonctions membranaires sont observables (Figure 7).

L'espace extracellulaire ne contient pas de matériel dense aux électrons, sauf autour

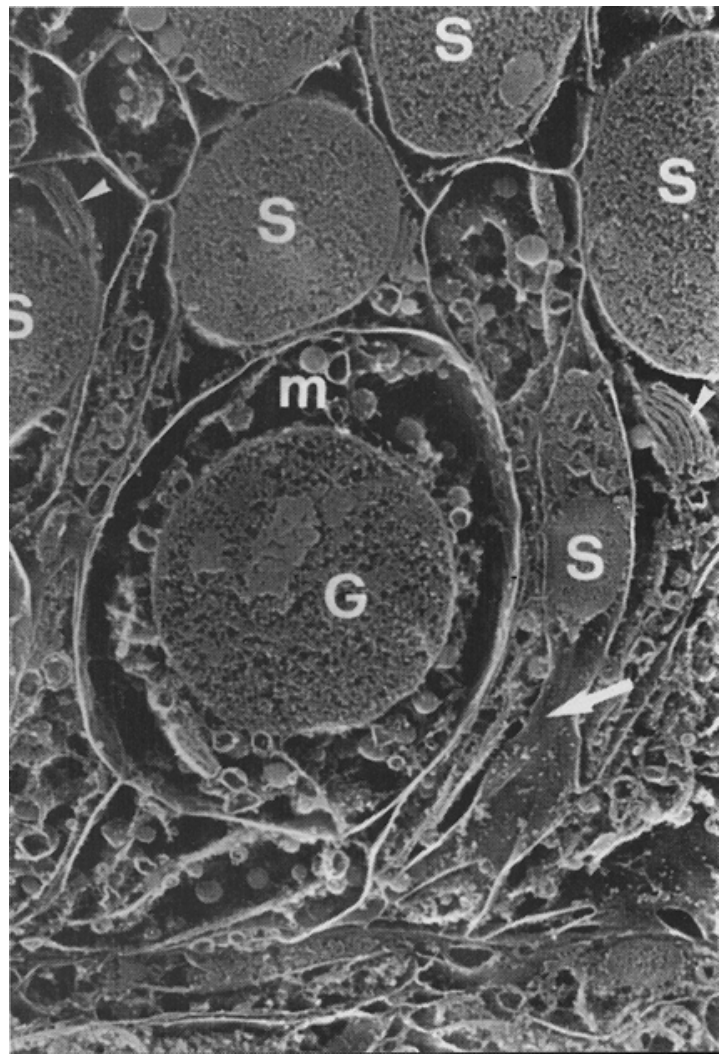

Figure 6 : Fracture d'un cordon testiculaire à 14 semaines de développement. Au centre une préspermatogonie (G). Des cellules présertoliennes (S) entourent complétement la préspermatogonie et montrent de longs processus cytoplasmiques (flèche) et des interdigitations. $m$ : mitochondries de la préspermatogonie. Têtes de flèches : reticulum endoplasmique granuleux des cellules présertoliennes. MEB+ODO, X 5000.

des cordons testiculaires là où une lamina basale s'est développée (Figures 4 et 7) [4, 17].

Entre 14 et 20 semaines de développement les noyaux des cellules présertoliennes sont pour la plupart disposés à proximité de la lamina basale et au dessus des préspermatogonies (Figures 3, 6 et 7). Leur cytoplasme contient des corps ronds denses de taille variable et le reticulum granuleux et les mitochondries sont observés surtout en localisation périnucléaire (Figures 6 et 7 ). Il 


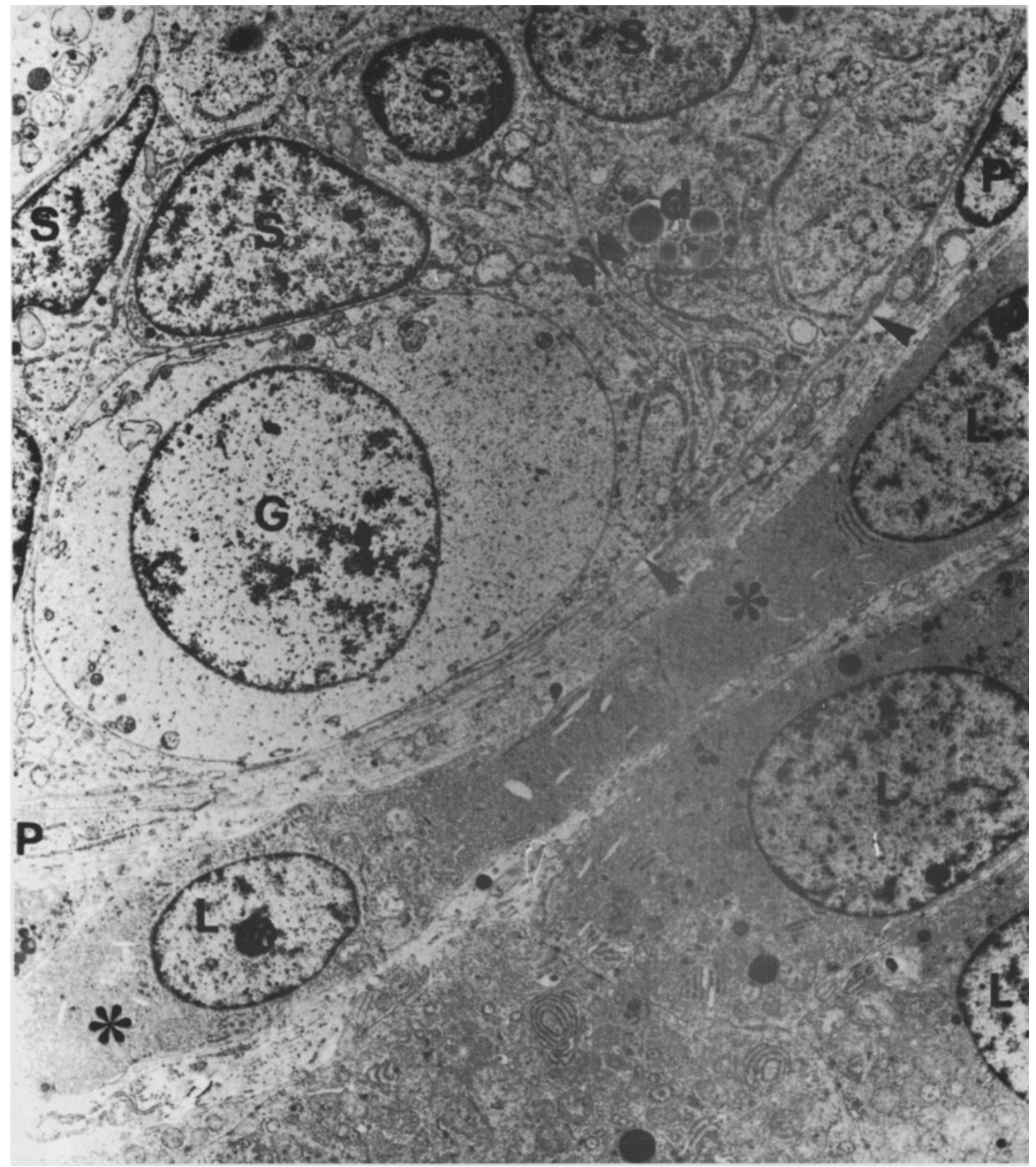

Figure 7 : Portion de cordon testiculaire dans un foetus humain à 16 semaines de développement. La coupe concerne une préspermatogonie (G) et plusieurs cellules présertoliennes (S). Les flèches larges indiquent un dispositif de jonction entre deux cellules présertoliennes voisines. $d:$ un groupe de corps denses (gouttelettes lipidiques) dans le cytoplasme d'une cellule présertolienne. Les têtes de flèches indiquent la lamina basale. P : future cellule péritubulaire. Dans la partie inférieure de la figure plusieurs cellules de Leydig différenciées $(L)$ montrent un arrangement épithélioüde et leur cytoplasme possède un abondant reticulum endoplasmique lisse (astérisque). MET, X 5000. 
est probable que le développement des cellules présertoliennes atteint un pic qui correspond chronologiquement au pic de sécrétion de testostérone et de MIS [2]. Les cordons testiculaires ne peuvent être qualifiés de "tubules" puisqu'il n'y a pas encore de lumière. Il est significatif que cette période soit également marquée par le développement maximum des cellules de Leydig dans l'interstitium (Figure 3 et 7). Ces cellules sont caractérisées par une plus grande densité aux électrons par rapport aux cellules mésenchymateuses, leurs noyaux arrondis, leurs volumineux nucléoles et surtout leur abondant reticulum endoplasmique lisse (Figure 7) [2, 10, 12].

Remerciements : Ce travail a été financé à $60 \%$ par MURST (1995-1996). Nous remercions le Pr. T.Naguro (Département d'Anatomie, Université Tottori, Yonago, Japon) pour son aide dans la préparation de certains échantillons (macération MET-ODO).

\section{REFERENCES}

1. BERGMANN M., KLIESCH S. : The distribution pattern of cytokeratin and vimentin immunoreactivity in testicular biopsies of infertile men. Anat. Embryol. Berl., 1994, 190 : 515-520.

2. BYSKOV A.G., HYER P.E. : Embryology of mammalian gonads and ducts. In : Knobil E., Neill J.D. eds. The physiology of reproduction, Second Edition. New-York, Raven Press, 1994 : 487-540.

3. CARREAU S., FOUCAULT P., DROSDOWSKY M.A. : La cellule de Sertoli. Aspects fonctionnels comparés chez le rat, le porc et l'homme. Ann. Endocrinol, Paris, 1994, 55 : 203-220.

4. FALIN L.I. : The development of genital glands and the origin of germ cells in human embryogenesis. Acta Anat., 1969, 72 : 195-232.

5. FUKUDA O., MIYAYAMA Y., FUJIMOTO T., OKAMURA H. : Electron microscopic study of the gonadal development in early human embryos. In : Motta P.M. ed. Developments in ultrastructure of reproduction. New York, Alan R. Liss, Inc, Progr. Clin. Biol. Res., 1989, 296 : 23-29.

6. GONDOS B., HOBEL C.J. : Ultrastructure of germ cell development in the human fetal testis ? Z. Zellforsch., 1971, $119: 1-20$.
7. HOANG-NGOC M., SMADJA A. : De l'embryologie de l'appareil genital feminin à la morphologie des états intersexués et des Malformations congénitales. Schering, Mame Tours, 1996.

8. JOSSO N. : Sexual differentiation. In: Adashi E.Y., Rock J.A., Rosenwaks Z. eds. Reproductive endocrinology, surgery, and technology. Vol. 1. Philadelphia, Lippincott-Raven Publishers, 1996 : 6074

9. LARSEN W.J. : Développement du système urogénital. In : Embryologie humaine. Bruxelles, De Boeck Université, 1996 : 235-279.

10. MAGRE S., JOST A. : Sertoli cells and testicular differentiation in the rat fetus. J.Electr. Microsc. Techn., 1991, $19: 172-188$.

11. MAKABE S., NAGURO T., MOTTA P.M. : A new approach to the study of ovarian follicles by scanning electron microscopy and ODO maceration. Arch. Histol. Cytol., 1992, 55: 183-190.

12. MAKABE S., NAGURO T., HEYN R., MOTTA P.M.: Ultrastructure of human Leydig cells at early gonadal embryogenesis. In : Motta P.M. ed. New trends in Microanatomy. Firenze, Ed. "II Sedicesimo". It. J. Anat. Embryol., 1995, 100 suppl. 1: 525-533.

13. MANCINI R.E., NARBAITZ R., LAVIERI J.C. : Origin and development of the germinative epithelium and Sertoli cells in the human testis ; cytological, cytochemical, and quantitative study. Anat. Rec., 1960, $136:$ 477-483.

14. MATSUMOTO A.M. : Spermatogenesis. In : Adashi E.Y., Rock J.A., Rosenwaks Z. eds. Reproductive endocrinology, surgery, and technology. Vol. 1. Philadelphia, Lippincott-Raven Publishers, 1996 : 359-382.

15. MAUDUIT C., BENAHMED M. : Growth factors in the testis development and function. In : Hamamah S., Mieusset R. eds. Research in male gametes : production and quality. France, INSERM, $1996:$ 3-44.

16. OSTRER H.: Sex determination. In : Adashi E.Y., Rock J.A. and Rosenwaks Z. eds. Reproductive endocrinology, surgery, and technology. Vol. 1. Philadelphia, Lippincott-Raven Publishers, 1996 : 42-58.

17. PELLINIEMI L.J., NIEMI M. : Fine structure of the human fetal testis. I. The interstitial tissue. $\mathrm{Z}$. Zellforsch., 1969, 99 : 507-522.

18. REY R., al-ATTAR L., LOUIS F., JAUBERT F., BARBET P., NIHOUL-FEKETE C. et al.: Testicular dysgenesis does not affect expression of antimullerian hormone by Sertoli cells in premeiotic seminiferous tubules. Am J. Pathol., 1996 148: $1689-1698$ 
19. ROGATSCH H., JEZEK D., HITTMAIR A., MIKUZ G., FEICHTINGER H.: Expression of vimentin, cytokeratin, and desmin in Sertoli cells of human fetal cryptorchid, and tumour-adjacent testicular tissue. Virchows Arch., 1996, 427 : 497 502.

20. SATOH M. : Histogenesis and organogenesis of the gonad in human embryos. J. Anat., 1991, 177 : 85107.

21. TANAKA K., NAGURO T. : High resolution scanning electron microscopy of cell organelles by a new specimen preparation method. Biomed. Res., 1981, 2, suppl. : 63-70.

22. VENDRELY E. : La descente testiculaire. Andrologie, $1995,5: 303-308$.

23. VON EBERHARD F.: Zur Frage der Sertoli'schen Zellen. Anat. Anz., 1936, 82 : 1-19.

24. VOSSMEYER J. : Zur Cytologie der pränatalen Gonaden-Entwicklung beim Menschen. I. Die Histogenese des Hodens, an Eponschnitten untersucht. Z. Anat. Enwickl.-Gesch., 1971, 134 : 146164.

25. WARTENBERG H., HOLSTEIN A.F., VOSSMEYER J. : Zur Cytologie der pränatalen Gonaden-Entwicklung beim Menschen. II. Elektronenmikroskopische Untersuchungen über die Cytogenese von Gonocyten und fetalen Spermatogonien im Hoden. Z. Anat. Entwickl.-Gesch., 1971, 134 : 165-185.

26. WARTENBERG H. : Human testicular development and the role of the mesonephros in the origin of a dual Sertoli cell system. Andrologia, 1978, 10 : $1-21$.

\section{ABSTRACT}

Ultrastructure of human Sertoli cells at early gonad embryogenesis

S. Makabe, R. Heyn, P.M. MotTa
The morphofunctional development of Sertoli cells defines the testicular differentiation. These somatic cells are mostly of mesonephric origin and can be first recognized in 7 week-old embryos altogether with the formation of testicular cords. The latter organize as primordial germ cells surrounded by pre-Sertoli cells. Due to the great synthetic activity of pre-Sertoli cells the rough endoplasmic reticulum develops. The basal lamina of the cords becomes distinguishable at 7 to 8 weeks of development. Either prespermatogonia and pre-Sertoli cells actively proliferate but the latter greatly outnumber prespermatogonia. Many interdigitations and cytoplasmic processes are observed between neighboring pre-Sertoli cells. Due to the proliferative activity a sort of compartmentalization is established inside the cords in which pre-Sertoli cells tend to localize closer to the basal membrane embracing the prespermatogonia with long and thin cytoplasmic processes. One of the main features typical of differentiating pre-Sertoli cells is the irregular nucleus and the prominent nucleolus. When the embryo is 14 to 20 weeks-old the most significative change is the maximum development of the Leydig cells. Testicular cords do not show lumen at all, so they cannot be termed "tubules"

Key words : Sertoli cells, testis, human embryogenesis, ultrastructure, SEM, TEM. 